\title{
Simulation of Compositional Variation in Liquid Phase Epitaxy InGaP Using a Two Dimensional Model
}

\author{
Hiromoto Susawa, ${ }^{\dagger}$ Toshiniro Tsuji,$^{\dagger}$ Kazumasa Hiramatsu,$^{\dagger \dagger}$ \\ TAKASHI JIMBO ${ }^{\dagger \dagger}$ and TetsuO SOGA ${ }^{\dagger}$
}

\begin{abstract}
Compositional variation in initial growth was observed in Liquid Phase Epitaxy (LPE) experiments. It is thought that the cause is the flow of the melt which is induced by the slide of the entire melt from the place where there isn't substrate to the substrate before growth. The phenomenon was simulated with a one dimensional model in InGaP growth with a convection term added to a diffusion-limited model. The velocity of flow in the melt was approximated to Stokes's first problem. It was shown that composition of In in grown solid with the flow is larger than that without the flow. In this paper, InGaP LPE growth is considered and a two dimensional model of the flow is used. For the solute transport, a two dimensional model is also adopted except at the growth interface. A result similar to the one dimensional model was obtained. In the two dimensional model, In composition is greater than that in the case without flow for the greater part of the growth time, but decreases when the flow transports dilute solution. This decrease doesn't appear in the one dimensional model. This phenomenon can be explained by considering the influence of the flow on the mole fraction near the boundary layer.
\end{abstract}

\section{Introduction}

Liquid Phase Epitaxy (LPE) is a method for growing crystals from liquid phase. It is used for optoelectronic devices. The LPE method has both advantages and disadvantages. One disadvantage is that it is difficult to control the composition of the epitaxial layer, which affects the fabrication of semiconductor lasers. In LPE, the growth condition at the growth interface is approximated to an equilibrium state between the solid phase and the liquid phase. It is possible to grow crystals with fewer defects by LPE when compared to epitaxies which grow under non-equilibrium methods, such as Vapor Phase Epitaxy. A calculation method to estimate the equilibrium condition was established ${ }^{1)}$ because it is important to calculate the equilibrium condition to estimate the mole fraction of the solid phase and the liquid phase.

In practice, LPE does not occur exactly at equilibrium. An example of the LPE experiment process is as follows:

(a) Sources which are solid are set in a crucible.

(b) The sources are melted and the liquid is

$\dagger$ Department of Environmental Technology and Urban Planning, Nagoya Institute of Technology

$\dagger \dagger$ Department of Electrical and Electronic Engineering, Mie University

$\dagger \dagger \dagger$ Research Center for Nano-Device and System, Nagoya Institute of Technology saturated at a certain temperature.

(c) The temperature is lowered and the liquid is supersaturated.

(d) The substrate contacts the liquid and crystal growth starts.

(e) The liquid is separated from the substrate and crystal growth ends.

Melt refers to melted material in the crucible. The part far from the growth interface is supersaturated during crystal growth. Therefore, in more precise simulations, the transport phenomena of materials are considered in addition to equilibrium at the growth interface. A diffusion-limited model was used to describe the transport phenomena in the simulation of $\mathrm{LPE}^{2)}$. The model considered supersaturation in the melt and simulated the thickness of the epitaxial layer well for growth times of several minutes. Without the model, we can't calculate the thickness of the epitaxial layer as mentioned above or the compositional variation as will be discussed later.

Compositional variation of the epitaxial layer in LPE was simulated several years later ${ }^{3), 4)}$. At the time, computers were 8 bits or 16 bits and slow and could barely calculate one point of the phase diagram. The diffusion-limited model couldn't be solved sufficiently for compositional variation. A method for solving a diffusionlimited model for compositional variation was proposed last year ${ }^{5)}$.

However, before crystal growth starts, the en- 


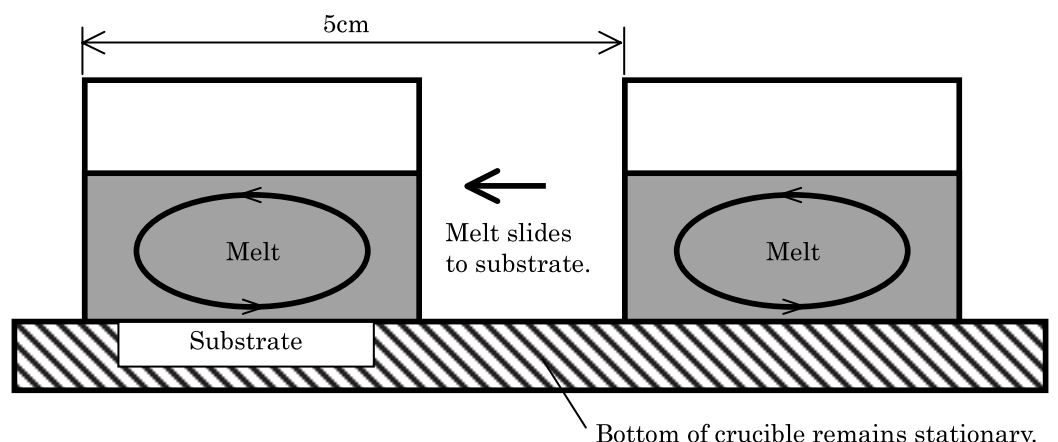

Fig. 1 Slide of the melt just before crystal growth.

tire melt slides from the place where there isn't substrate to the substrate. The process induces flow in the melt. It was reported that for short growth times, convection affects growth ${ }^{6}$. A convection term must be added to the diffusionlimited model to describe compositional variation. It was calculated that the convection affects compositional variation ${ }^{7)}$. In that report, the calculation model of flow was a one dimensional model. The convection term was roughly approximated and the diffusion equations were one dimensional. There was a case in which the results were within error in traditional calculations of compositional variation as mentioned above and pointed out in Ref. 5). But the result of this one dimensional model with an added convection term wasn't within error. We could detect compositional variation. The one dimensional model treated InGaP LPE growth. It was shown that composition of In in grown InGaP with flow in the melt was larger than without flow initially. This point is consistent with experiments ${ }^{4)}$.

Actually, at least one vortex exists in the melt, and its center moves ${ }^{6}$. . Accordingly, there is a flow perpendicular to the growth interface near the vortex. But the flow perpendicular to the growth interface in the melt isn't considered in a one dimensional model. Therefore, a two dimensional model is necessary to consider this flow. In this paper, we use a two dimensional model for the flow in the melt. A two dimensional model is also adopted for the transport of solute except at the growth interface. This paper shows a phenomenon similar to the one dimensional model and also a phenomenon which doesn't appear in the one dimensional model.

\section{Calculation Model}

\subsection{Experiment to Analyze}

In this paper, the LPE experiment in Ref. 4) is analyzed. The substrate is GaAs, which is a III-V binary compound. The crystal grown on the substrate is InGaP which is a III-V alloy. The melt consists of In, P and Ga. The solvent is In. The solutes are $\mathrm{P}$ and Ga.

\subsection{Thermodynamic Model}

Liquid phase and solid phase at the growth interface were treated as equilibrium states. The thermodynamic model at the growth interface is the same as in Ref.4). This paper considers transport phenomena. The phenomena that occur one second after crystal growth starts are examined. Therefore, temperature is approximated as a constant during the growth. A growth temperature of 782 degrees is adopted in Ref. 4).

\subsection{Flow in The Melt}

In the LPE process, the melt is supersaturated where there is no substrate. Then the entire melt slides to the substrate to grow crystal on the substrate. The bottom which the melt touches remains stationary. Accordingly, the slide induces flow in the melt. The distance of the slide is $5 \mathrm{~cm}$ as illustrated in Fig. $\mathbf{1}$.

\subsection{Melt Slide to Substrate Model}

Set the velocity of the melt's slide just before the growth to $U_{0}=20 \mathrm{~cm} / \mathrm{s}$. Then the melt stops on the substrate. In the calculation model, it is represented as follows:

(a) Initially, the melt is stationary.

(b) Next, the bottom of the melt slides at a speed of $U_{0}=20 \mathrm{~cm} / \mathrm{s}$ for time $t_{0}=$ (the distance of the slide $5 \mathrm{~cm}) / U_{0}$, and the sides of the melt remain stationary.

(c) The velocity of the bottom is set to 0 .

We consider the two dimensional effect of the flow and diffusion equations in addition to the 


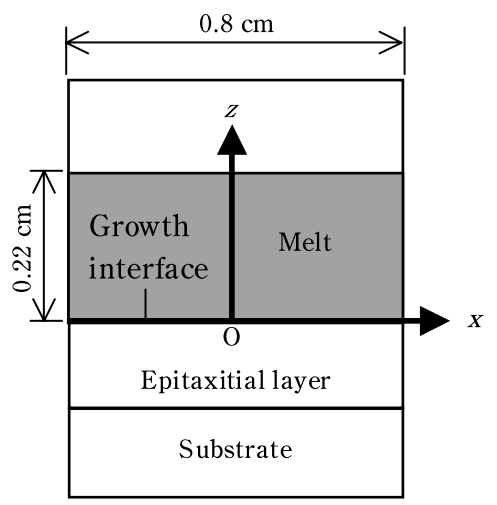

Fig. 2 Coordinate system.

one dimensional effect ${ }^{4), 7)}$. The coordinate system is shown in Fig. 2. $x$ is the coordinate in the opposite direction of the slide of the entire melt to the substrate. $z$ is the coordinate from the growth interface.

The size of the melt used in the calculation is the actual size in the experiment ${ }^{8), 9)}$. The size of the melt is $0.8 \mathrm{~cm}$ in the direction of $x$ and $0.22 \mathrm{~cm}$ in the direction of $z$. The origin of the coordinate system is located at the midpoint of the growth interface.

\subsection{Review of The One Dimensional Model ${ }^{7)}$}

The one dimensional model treated LPE growth of InGaP and calculated compositional variation of solid phase. Forced convection induced by the slide of the entire melt was considered. Velocity of flow in the melt is approximated to the analytic solution of Stokes's first problem ${ }^{6)}$. The $z$ component of velocity $w$ is neglected. The $x$ component of velocity $u$ is given as follows.

$$
\begin{gathered}
u=U_{0}\left\{\operatorname{erfc}\left(\frac{z}{2 \sqrt{\nu\left(t_{0}+t\right)}}\right)\right. \\
\left.-\operatorname{erfc}\left(\frac{z}{2 \sqrt{\nu t}}\right)\right\},
\end{gathered}
$$

where $\nu$ is the kinematic viscosity of the melt. The melt consists of In whose mole fraction is greater than 90 percent. Then $\nu$ is the value of In and $1.7 \mathrm{E}-3 \mathrm{~cm}^{2} / \mathrm{s}^{9), 10)}$.

A diffusion-limited model ${ }^{2), 3)}$ was adopted to describe transport phenomena of $\mathrm{P}$ and Ga solutes. Diffusion in the direction of $x$ was neglected. Convection in the direction of $x$ was considered. Convection in the direction of $z$ was neglected. The simplified convection term was added to the diffusion equation.

$$
\begin{aligned}
& \frac{\partial X_{i}^{l}(x=0, z, t)}{\partial t} \\
& =-u \frac{2}{L}\left(\left(X_{i}^{l}(0, \infty, t)-\left(X_{i}^{l}(0, z, t)\right)\right.\right. \\
& \quad+D_{i} \frac{\partial^{2} X_{i}^{l}(0, z, t)}{\partial z^{2}}, \\
& \frac{\frac{1}{2}(1-a) r-X_{G a}^{l}(0,0, t)}{\frac{1}{2} r-X_{P}^{l}(0,0, t)} \\
& =\frac{\left.D_{G a} \frac{\partial X_{G a}^{l}(0, z, t)}{\partial z}\right|_{z=0}}{\left.D_{P} \frac{\partial X_{P}^{l}(0, z, t)}{\partial z}\right|_{z=0}}
\end{aligned}
$$

where $L$ is the size of the melt in the direction of $x . a$ is the composition of solid $\mathrm{In}_{a} \mathrm{Ga}_{1-a} \mathrm{P}$. $X_{i}^{l}(x, z, t)$ is the mole fraction of component $i$ in the melt at position $x, z$ and growth time $t$. Component $i$ represents $G a$ and $P . D_{i}$ is the diffusion coefficient of component $i . D_{G a} / D_{P}$ $=0.56$. The value was used in Ref.4) to fit results of the simulation to the experimental results. $D_{P}=1.6 \mathrm{E}-4 \mathrm{~cm}^{2} / \mathrm{s}$ so that calculated growth thickness is fit to experimental results. Equations represented by Eq. (2) for $i=G a$ and $P$ were solved by finite difference method explicitly. It is solved implicitly to satisfy the thermodynamic model and Eq. (3). Mesh size in the direction of $z$ is $6.6 \mathrm{E}-5 \mathrm{~cm}$ to detect compositional variation of solid phase at $t=0.01 \mathrm{~s}$. Time interval $\Delta t$ is set so that Eq. (2) is solved stably. The numerical calculation method was described in Ref. 5).

\subsection{Calculation of The Two Dimen- sional Flow}

When calculating the flow in the melt, the melt is treated as liquid In and incompressible because the In mole fraction of the melt is greater than 90 percent. The viscosity is constant. The kinematic viscosity of the melt $\nu$ is $1.7 \mathrm{E}-3 \mathrm{~cm}^{2} / \mathrm{s}^{9), 10)}$. It is the same as in the one dimensional model.

In the two dimensional model, the $z$ component of velocity $w$ is considered.

The basic equations are as follows:

The conservation of mass

$$
\frac{\partial u}{\partial x}+\frac{\partial w}{\partial z}=0
$$

Navier-Stokes equations

$$
\begin{aligned}
\frac{\partial u}{\partial t} & =-u \frac{\partial u}{\partial x}-w \frac{\partial u}{\partial z}-\frac{1}{\rho} \frac{\partial p}{\partial x} \\
& +\nu\left(\frac{\partial^{2} u}{\partial x^{2}}+\frac{\partial^{2} u}{\partial z^{2}}\right)
\end{aligned}
$$




$$
\begin{aligned}
\frac{\partial w}{\partial t} & =-u \frac{\partial w}{\partial x}-w \frac{\partial w}{\partial z}-\frac{1}{\rho} \frac{\partial p}{\partial z} \\
& +\nu\left(\frac{\partial^{2} w}{\partial x^{2}}+\frac{\partial^{2} w}{\partial z^{2}}\right),
\end{aligned}
$$

where $\rho$ is the density of the melt and $p$ is pressure. These equations were solved numerically with SIMPLE ${ }^{11)}$. The mesh size in the direction of $x$ is $2.9 \mathrm{E}-3 \mathrm{~cm}$. The mesh size in the direction of $z$ is $1.4 \mathrm{E}-3 \mathrm{~cm}$. The time interval $\Delta t$ is set so that the Courant number is less than 0.5 .

\subsection{Transport of Solutes in The Melt}

The diffusion-limited model is widely used for simulation of LPE as described in the introduction. The model under consideration for the transport of solutes is a diffusion-limited model with a convection terms added. The basic equation is as follows:

$$
\begin{aligned}
& \frac{\partial X_{i}^{l}(x, z, t)}{\partial t} \\
& =-u \frac{\partial X_{i}^{l}(x, z, t)}{\partial x}-w \frac{\partial X_{i}^{l}(x, z, t)}{\partial z} \\
& \quad+D_{i} \frac{\partial^{2} X_{i}^{l}(x, z, t)}{\partial x^{2}} \\
& \quad+D_{i} \frac{\partial^{2} X_{i}^{l}(x, z, t)}{\partial z^{2}}
\end{aligned}
$$

This paper considers the differences between the one dimensional and two dimensional models. The variation of the mole fraction of liquid phase at the growth interface is smaller than the amount of supersaturation. It is less than 10 percent of the supersaturation. Therefore, the boundary condition at the growth interface was evaluated only at $x=0$. The boundary condition is Eq. (3). The solutions are substituted into $X_{i}^{l}(x, z=0, t)$ and In composition of $\operatorname{In}_{a} \mathrm{Ga}_{1-a} \mathrm{P} a$ all over the growth interface. Equations represented by Eq. (7) for $i=G a$ and $P$ were solved by the finite difference method using $u$ and $w$ solved from Eqs. (4), (5) and (6) explicitly. The mesh size in the direction of $x$ is the same as that used to calculate the flow in the melt. The mesh size in the direction of $z$ is the same as in the one dimensional model described in the subsection above. The time interval $\Delta t$ is set so that Eq. (7) is solved stably ${ }^{12)}$. Mesh sizes in the directions of $z$ and $\Delta t$ are much smaller than those used to solve the flow. Therefore, $u$ and $w$ were interpolated by the cubic spline for $z$ direction and linearly for time. The segmentation of the melt in front of the growth interface is shown in Fig. 3.

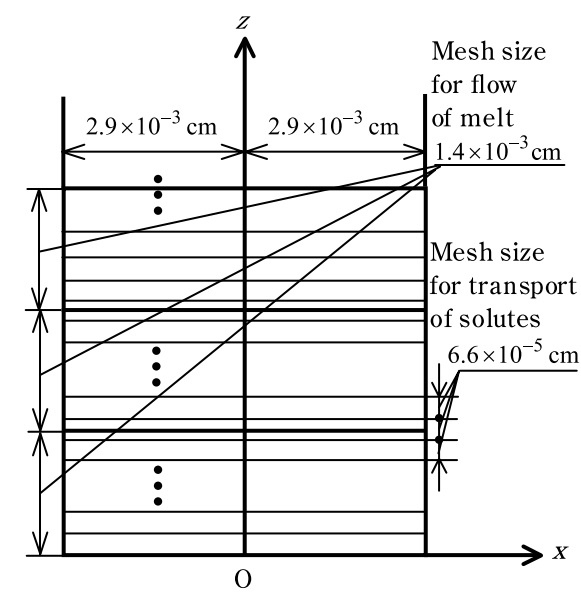

Fig. 3 Segmentation of the melt in front of the growth interface.

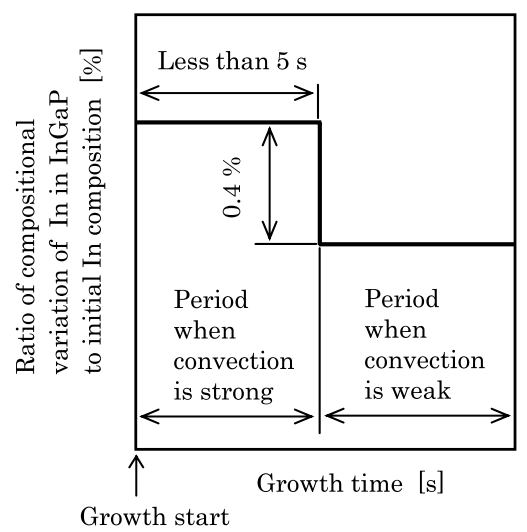

Fig. 4 Schematic illustration of compositional variation in the experiment.

\section{Results}

In the experiment ${ }^{4)}$, a solid InGaP of high In composition grew for the first 5 seconds after growth start. It is thought that the convection of the flow in the melt was strong for that period. After that period, solid InGaP of small In composition grew for the period when it is thought that the convection of the flow in the melt was weak. The ratio of compositional variation of In in InGaP to the initial In composition between large In composition and small In composition was 0.4 percent. Figure 4 illustrates the experiment schematically.

In the one dimensional calculation ${ }^{7)}$, it was shown that composition of In in grown InGaP with flow in the melt was larger than without flow initially. A similar result was obtained in the two dimensional calculation. Moreover, complicated structure exists. For exam- 
ple, composition of In in solid phase with flow in the melt is smaller than that in the case of no flow of the melt when the growth time was between 0.105 seconds and 0.259 seconds. Figure 5 shows the results, where 0 on the vertical axis corresponds to In composition in the case without flow of the melt.

The complicated structure is caused by the flow in the melt. When the flow transports con-

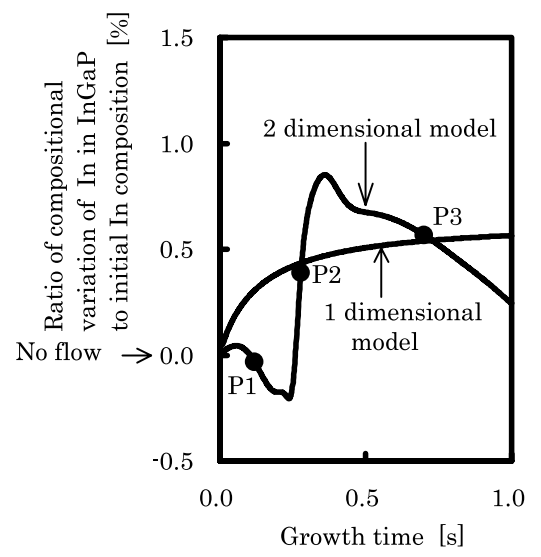

Fig. 5 Compositional variation of In in InGaP. centrated solution, boundary layers are filled with solutes ${ }^{7)}$. When the flow transports dilute solution, the opposite phenomenon occurs.

Figures 6, 7, 8, 9, 10, and 11 show the velocity vector of the flow in the melt. A large vortex exists just before crystal growth starts (Fig. 6). The vortex is induced by the slide of the entire melt to the substrate. A similar vortex was observed in an experiment of flow visualization using water and dye ${ }^{6)}$.

\section{Discussion}

In Fig. 5, composition of In in InGaP is increased by the flow of the melt initially. The composition is decreased at point P1 when growth time is 0.12 seconds. A vortex is generated near the midpoint of the growth interface (Fig. 7). The fluid around the vortex flows from the growth interface where the solution is dilute and upward near the midpoint of the growth interface. Let's try to understand why the flow causes a decrease of In composition in the solid phase intuitively.

\subsection{Supersaturation}

First, supersaturation is considered. $\Delta X_{i}^{l}$

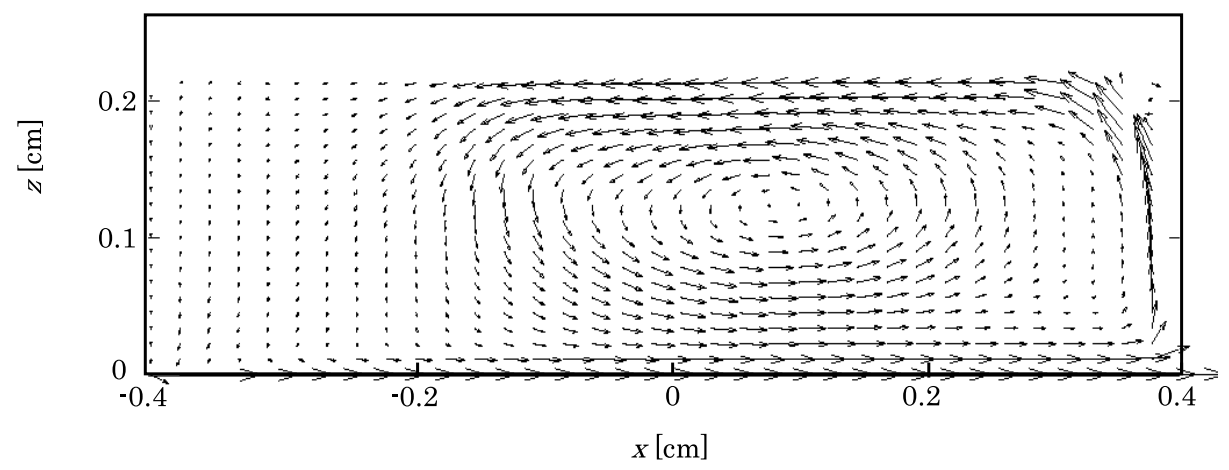

Fig. 6 Velocity vector of the flow in the melt just before growth.

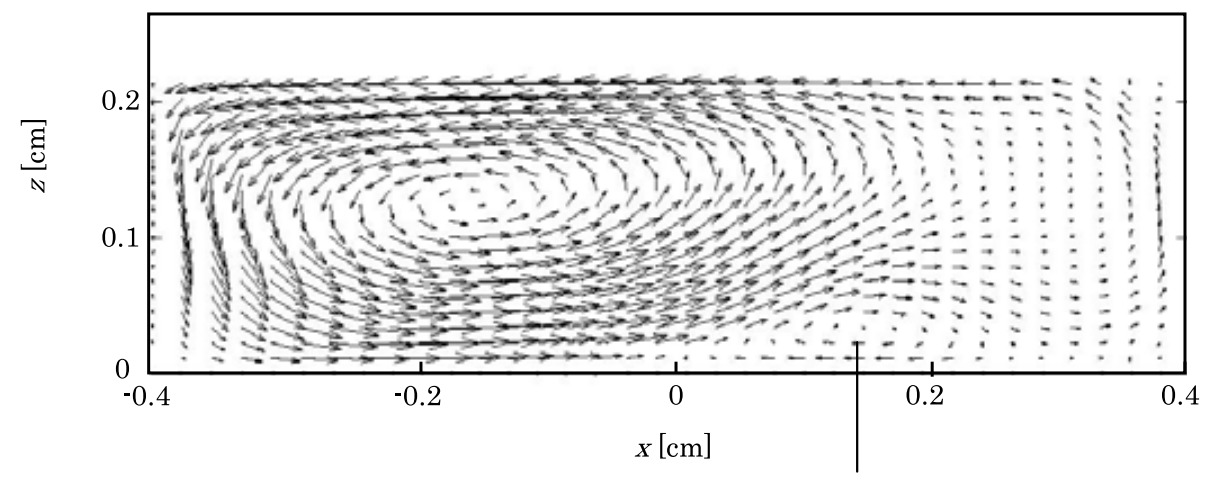

Generated vortex affects the composition of solid.

Fig. 7 Velocity vector of the flow in the melt at growth time 0.12 seconds. 


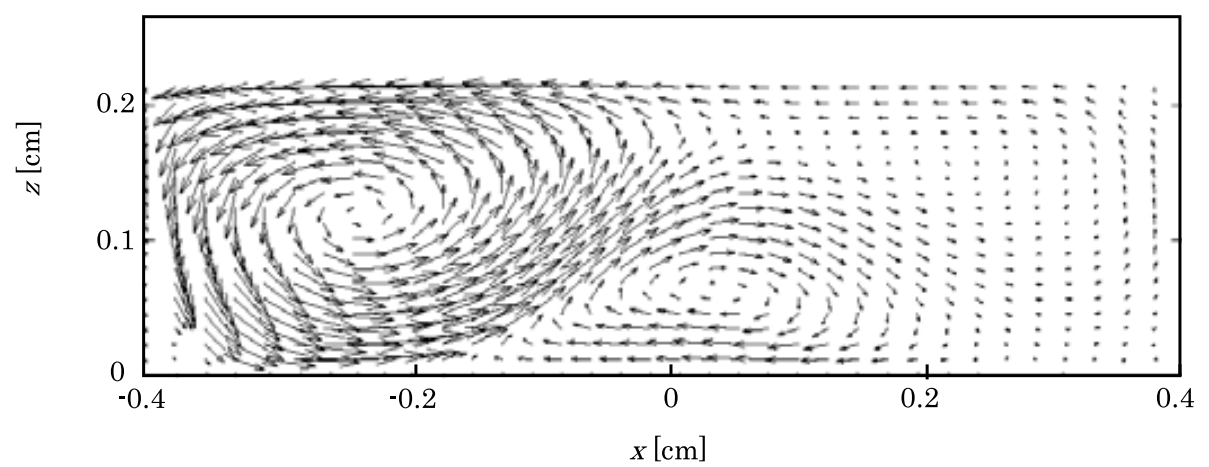

Fig. 8 Velocity vector of the flow in the melt at growth time 0.2 seconds.

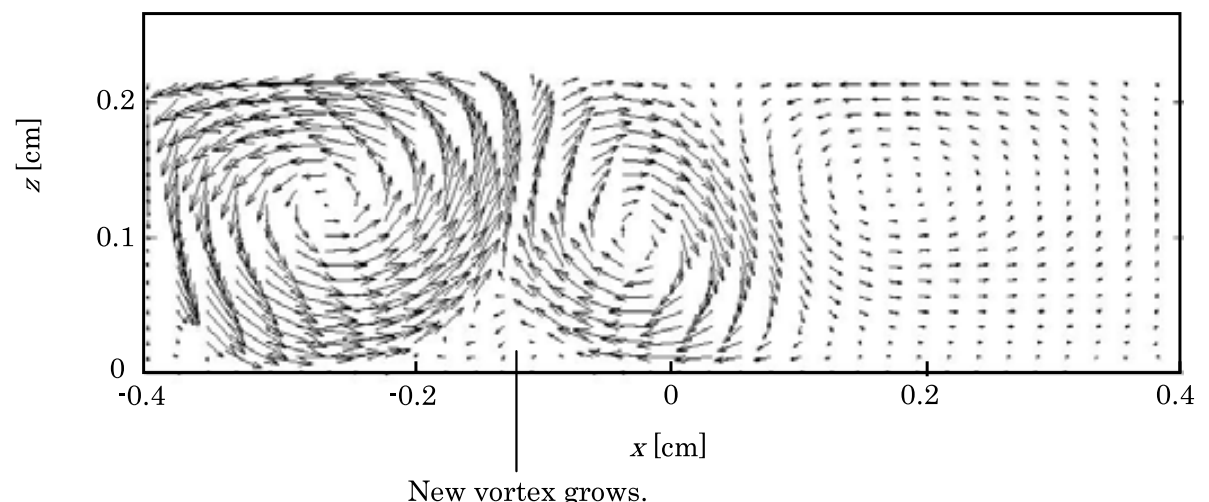

Fig. 9 Velocity vector of the flow in the melt at growth time 0.278 seconds.

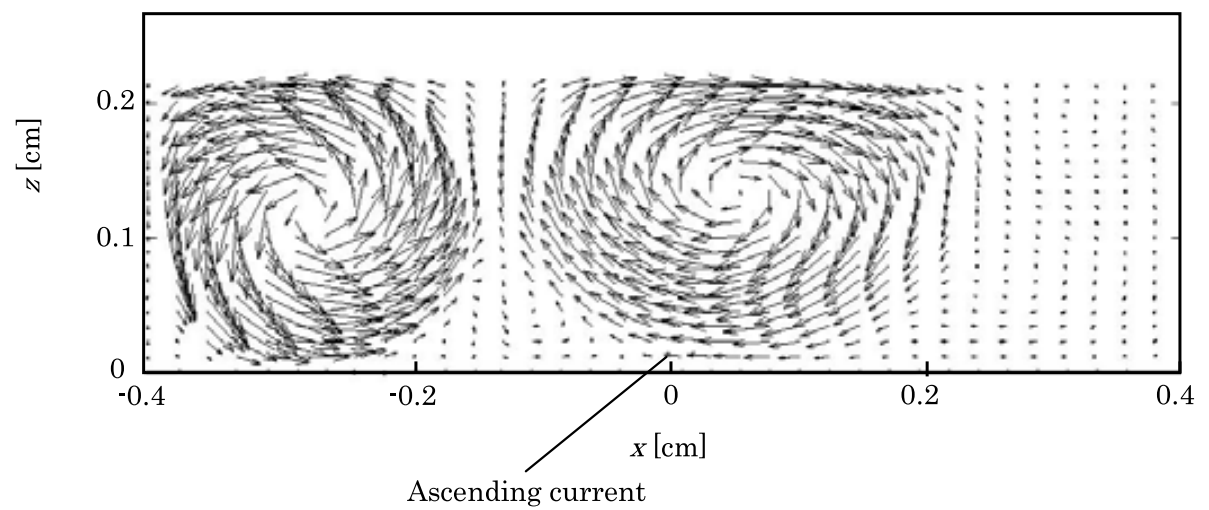

Fig. 10 Velocity vector of the flow in the melt at growth time 0.7 seconds.

stands for supersaturation of component $i$ at $x=0$.

$$
\Delta X_{i}^{l}=X_{i}^{l}(0, z, t)-X_{i}^{l}(0,0, t) .
$$

Figure 12 shows $\Delta X_{i}^{l}$ at point P1 in Fig. 5 growth time 0.12 seconds. The mole fraction in liquid phase remains at its initial value at $t=0$ further away from the growth interface. Crystal growth consumes solutes in the melt. Then the mole fraction of the solutes near the growth interface decreases. The part where the mole fraction decreased is called the boundary layer. The boundary layer thickness is defined in Fig. 13.

The boundary layer thickness is $1.6 \times 10^{-2}$ $\mathrm{cm}$ for $\mathrm{P}$ in Fig. 12. In this calculation, the method of LPE growth is called step-cooling. When there is no flow in melt, boundary layer thickness is calculated from analytic solution of step-cooling in Ref.13). Then the bound- 


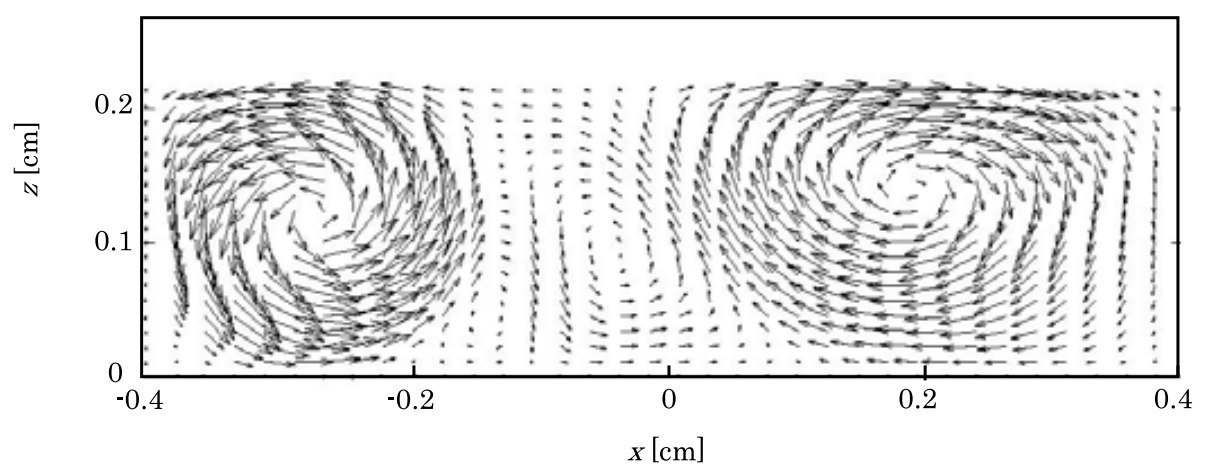

Fig. 11 Velocity vector of the flow in the melt at growth time 1 second.

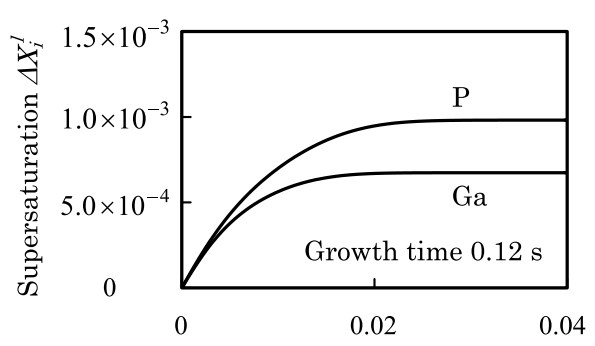

Height from the growth interface [cm]

Fig. 12 Supersaturation at growth time 0.12 seconds.

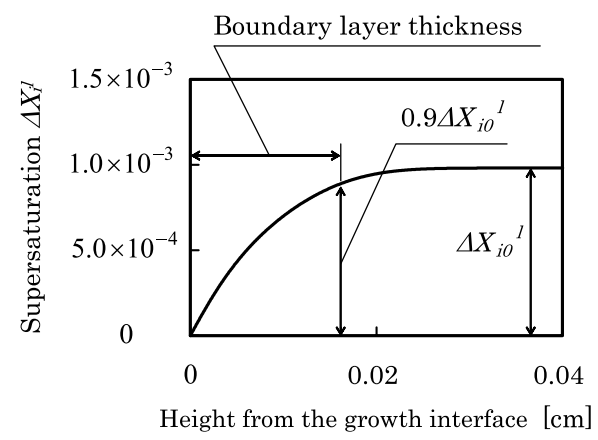

Fig. 13 Definition of boundary layer thickness.

ary layer thickness is $1.1 \times 10^{-2} \mathrm{~cm}$ for $\mathrm{P}$ at $t=0.12$ seconds in the case of no flow. Accordingly, the boundary layer thickness in Fig. 12 is similar to the case with no flow in the melt. $\Delta X_{i 0}^{l}$ is defined in Fig. 13. $\Delta X_{i}^{l}$ is constant and $\Delta X_{i}^{l}=\Delta X_{i 0}^{l}$ where $z$ is large enough. Therefore, the main reason why $\Delta X_{i}^{l}$ is constant outside of the boundary layer is not that flow in the melt transports solutes to the part where solutes are consumed but rather that the decrease of the consumption of solutes does not reach the part where $\Delta X_{i}^{l}$ is constant.

The features of Fig. 12 are as follows:

- The boundary layer of $\mathrm{P}$ is larger than that of Ga.
- $\Delta X_{P 0}^{l}>\Delta X_{G a 0}^{l}$

- The first-order partial derivative of $\Delta X_{i}^{l}$ with respect to $z$ at the growth interface for $\mathrm{P}$ is almost the same as that for Ga.

Applying the above features, the influence of flow in the melt on supersaturation is discussed.

\subsection{Influence of Flow in The Melt on Supersaturation}

Now, we shall discuss the reason why the flow causes a decrease of In composition in the solid phase. Equation (3) determines composition of solid phase. In the left side of the equation, $a$ is about 0.5 and $r$ is on the order of $1 . X_{G a}^{l}$ and $X_{P}^{l}$ are on the order of 0.01 . Therefore, the left side is approximated to $1-a$. Finally, Eq. (3) is approximated as follows.

$$
\begin{aligned}
& 1-a \\
& \approx \frac{\left.D_{G a} \frac{\partial X_{G a}^{l}(0, z, t)}{\partial z}\right|_{z=0}}{\left.D_{P} \frac{\partial X_{P}^{l}(0, z, t)}{\partial z}\right|_{z=0}}
\end{aligned}
$$

Then composition of the solid strongly depends on the first-order partial derivative of $X_{i}^{l}$ with respect to $z$ at the growth interface.

Using the feature of Fig. 12 discussed above, Fig. 12 is simplified to Fig. 14. In Fig. 14, it is assumed that the solution at point Z1 is transported to point Z2. The solution at point Z1 is more dilute than that at point Z2. Then, a decrease of $\mathrm{P}$ is larger than that of Ga. Accordingly, the decrease in the first-order partial derivative of $X_{i}^{l}$ with respect to $z$ at the growth interface for $\mathrm{P}$ is larger than that for Ga. This causes an increase of $1-a$ from Eq. (9).

Therefore, composition of In in solid phase decreases because of the flow which transports dilute solution.

\subsection{Verification of Behavior at Point $\mathbf{P 1}$ in Fig. 5}

In Fig. 5, the composition is decreased at 


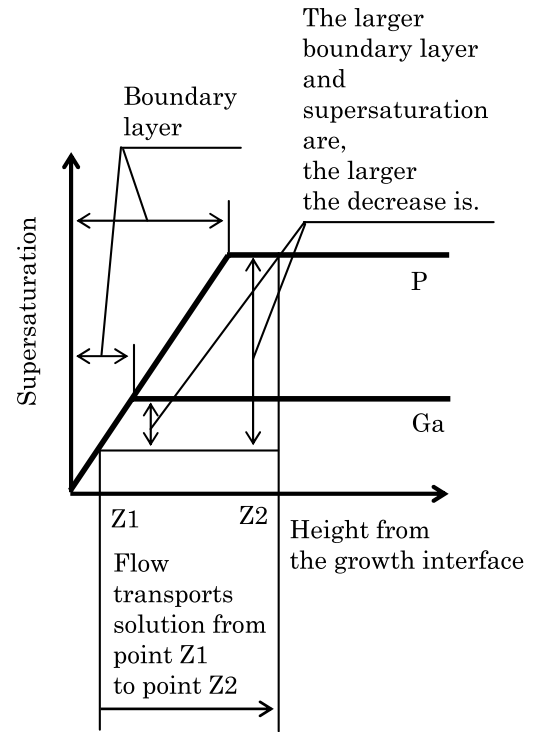

Fig. 14 Influence of convection on supersaturation.

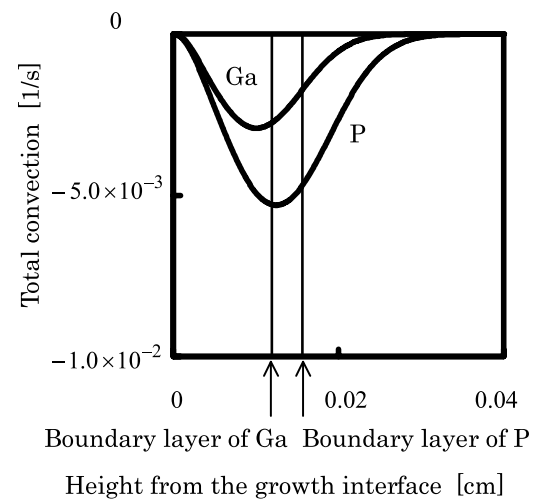

Fig. 15 Total convection at growth time 0.12 seconds.

point $\mathrm{P} 1$ at a growth time of 0.12 seconds. The solution flows upward near the mid point of the growth interface in Fig. 7. The solution is dilute near the growth interface. It is found that the decrease is caused by the flow from the above discussion.

Let us evaluate the amount of convection at this growth time. Figure $\mathbf{1 5}$ shows this value near the growth interface at $x=0$. The convection decreases the mole fraction near the boundary layer. The total convection ratio of $\mathrm{P}$ to $\mathrm{Ga}$ is as follows.

(Total convection ratio of $\mathrm{P}$ to $\mathrm{Ga}$ )

$$
=\frac{-u \frac{\partial X_{P}^{l}(x, z, t)}{\partial x}-w \frac{\partial X_{P}^{l}(x, z, t)}{\partial z}}{-u \frac{\partial X_{G a}^{l}(x, z, t)}{\partial x}-w \frac{\partial X_{G a}^{l}(x, z, t)}{\partial z}} .
$$

The total convection ratio of $\mathrm{P}$ to Ga per su-

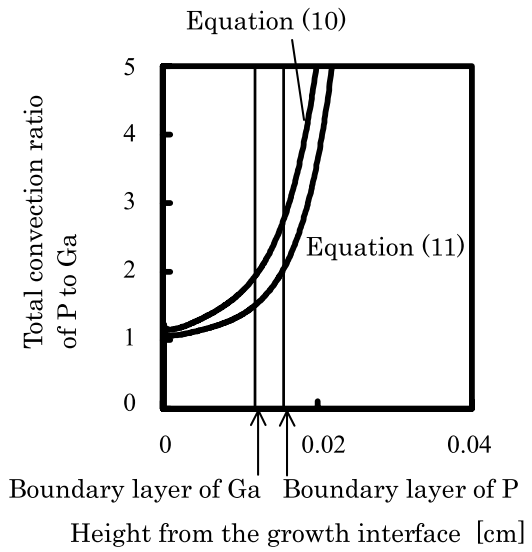

Fig. 16 Total convection ratio of $\mathrm{P}$ to $\mathrm{Ga}$ at growth time 0.12 seconds.

persaturation is as follows:

(Total convection ratio of $\mathrm{P}$ to $\mathrm{Ga}$ )

$$
\times \frac{X_{G a}^{l}(0, z, t)-X_{G a}^{l}(0,0, t)}{X_{P}^{l}(0, z, t)-X_{P}^{l}(0,0, t)} .
$$

These values are shown in Fig. 16. The decrease of $\mathrm{P}$ by convection is greater than that of Ga.

\subsection{Behavior at Point P2 and P3 in Fig. 5}

In Fig. 5, composition of In in InGaP is increased at point $\mathrm{P} 2$ at growth time 0.278 seconds. The velocity vector of the flow in the melt is shown in Fig. 9. The flow transports concentrated solution to the midpoint of the growth interface. The increase of In in In$\mathrm{GaP}$ is understood with similar considerations described above.

In Fig. 5, composition of In in InGaP is decreased at point $\mathrm{P} 3$ at growth time 0.7 seconds. The velocity vector of the flow in the melt is shown in Fig. 10. The melt flows upward at the midpoint of the growth interface. The flow decreases composition of In in solid InGaP for the same reason as the decrease of In at point $\mathrm{P} 1$ in Fig. 5 .

\subsection{Influence of Large Melt Size in The Direction of $x$}

It is thought that the substrate is large in mass production. Then the size of the melt in the direction of $x$ is larger than that in the calculation of this paper. If the size of the melt in the direction of $x$ becomes large, the two dimensional effect becomes weak. Even in that case, there are vortices in the melt. The two dimensional effects, such as are shown in the calculation of this paper, affect the composition 
of the solid phase.

\section{Conclusions}

Compositional variation of solid InGaP is calculated with a two dimensional model of the flow in the melt and the transport of solutes except for the boundary condition at the growth interface in LPE. The size of the melt used in the calculation is the actual size used in the experiment.

The composition of In in a grown solid with flow in the melt was larger than without flow of the melt initially. This point is consistent with experiments and the one dimensional model.

In the two dimensional model, composition of In was greater than that in the case of no flow at the greater part of the growth time, but decreased when the flow transported dilute solution. The decrease does not appear in the one dimensional model. This phenomenon is understood by considering the influence of the flow on the mole fraction near the boundary layer.

It is thought that the substrate is large in mass production. Then the size of the melt in the direction of $x$ is larger than that in the calculation of this paper. If the size of melt in the direction of $x$ becomes large, the two dimensional effect becomes weak. Even in that case, there are vortices in the melt. The two dimensional effect, such as is shown in the calculation of this paper, affects the composition of the solid phase.

Acknowledgments This work was partly supported by the NITECH 21st Century COE Program.

\section{References}

1) Ilegems, M. and Panish, M.B.: Phase Equilibria in III-V Quaternary Systems-Application to Al-Ga-As, J. Phys. Chem. Solids, Vol.35, pp.409-420 (1974).

2) Crossley, I. and Small, M.B.: Computer Simulations of Liquid Phase Epitaxy of GaAs in Ga Solution, J. Cryst. Growth, Vol.11, pp.157-165 (1971).

3) Ijuin, H. and Gonda, S.: Computer Simulations of Liquid Phase Epitaxy of III-V Ternary Alloys, J. Cryst. Growth, Vol.33, pp.215-222 (1976).

4) Hiramatsu, K., Tanaka, S., Sawaki, N. and Akasaki, I.: Analysis of Compositional Variation at Initial Transient Time in LPE Growth of InGaAsP/GaAs System, Jpn. J. Appl. Phys., Vol.24, No.8, pp.1030-1035 (1985).

5) Susawa, H., Tsuji, T., Jimbo, T. and Soga,
T.: Initial Condition and Calculation Method in Numerical Simulation of LPE, J. Chem. Eng. Japan.

6) Leung, S.Y. and Schumaker, N.E.: Simulation of Slider-Induced Convection in Horizontal LPE Slider system, J. Cryst. Growth, Vol.60, pp.421-433 (1982).

7) Susawa, H., Tsuji, T., Hiramatsu, K., Jimbo, T. and Soga, T.: Simulation of InGaP Liquid Phase Epitaxy Including Convection, Theoretical and Applied Mechanics Japan, Vol.55, pp.279-284 (2006).

8) Hiramatsu, K.: Research in Liquid Phase Epitaxy of InGaAsP/GaAs Alloy Semiconductors, Ph.D. Thesis, Nagoya University (1986).

9) Lide, D.R. and Frenderikse, H.P.R. (Ed.): Handbook of Chemistry and Physics, CRC Press Corp., 76th ed., pp.4-125, Boca Raton, New York, London and Tokyo (1995).

10) Cheng, S., Bian, X., Zhang, J., Qin, X. and Wang, Z.: Correlation of viscosity and structural changes of indium melt, Materials Letters, Vol.57, pp.4191-4195 (2003).

11) Patankar, S.V.: Numerical Heat Transfer and Fluid Flow, Hemisphere Publishing Corp., Washington, D.C. (1980).

12) Roache, P.J.: Computational Fluid Dynamics, Hermosa Publishers Inc., Albuquerque, N.M. (1976)

13) Feng, M., Cook, L.W., Tashima, M.M. and Stillman, G.E.: Lattice Constant, Bandgap, Thickness, and Surface Morphology of InGaAsP-InP Layers Grown by Step-Cooling, Equilibrium-Cooling, Supercooling and TwoPhase-Solution Growth Techniques, J. Electronic Materials, Vol.9, No.2, pp.241-280 (1980).

\section{Appendix: Notation}

\begin{tabular}{|c|c|}
\hline & \\
\hline & $\begin{array}{l}\text { opposite direction of the } \\
\text { slide of the melt to } \\
\text { the substrate }\end{array}$ \\
\hline & $\begin{array}{l}: \text { the coordinate from } \\
\text { the growth interface }\end{array}$ \\
\hline & : growth time \\
\hline$\iota$ & : $x$ component of velocity \\
\hline$w$ & $: z$ component of velocity \\
\hline & : pressure \\
\hline$X_{i}^{l}(x, z, t)$ & $\begin{array}{l}: \text { the mole fraction of } \\
\text { component } i \text { in the melt } \\
\text { at positions } x, z \text { and } \\
\text { growth time } t\end{array}$ \\
\hline & $\begin{array}{l}: \text { the kinematic viscosity } \\
\text { of the melt }\end{array}$ \\
\hline & the density of the melt \\
\hline
\end{tabular}




\begin{tabular}{|c|c|}
\hline$D_{i}$ & $\begin{array}{l}: \text { the diffusion coefficient } \\
\text { of component } i\end{array}$ \\
\hline & in the melt \\
\hline$r$ & $\begin{array}{l}: \text { the ratio of the volume } \\
\text { per unit atom in the }\end{array}$ \\
\hline & $\begin{array}{l}\text { liquid to that in the } \\
\text { solid }\end{array}$ \\
\hline$a$ & $\begin{array}{l}: \text { the composition of In } \\
\text { in solid } \operatorname{In}_{a} \mathrm{Ga}_{1-a} \mathrm{P}\end{array}$ \\
\hline$L$ & $\begin{array}{l}: \text { the size of the melt in the } \\
\text { direction of } x\end{array}$ \\
\hline$U_{0}$ & $\begin{array}{l}: \text { the velocity of the melt } \\
\text { which slides to } \\
\text { the substrate }\end{array}$ \\
\hline$t_{0}$ & $\begin{array}{l}: \text { the time for which the melt } \\
\text { slides to the substrate }\end{array}$ \\
\hline$\Delta X_{i}^{l}$ & $\begin{array}{l}: \text { supersaturation of } \\
\text { component } i \text { at } x=0 \\
\text { in the melt }\end{array}$ \\
\hline$\Delta X_{i 0}^{l}$ & $\begin{array}{l}: \Delta X_{i 0}^{l} \text { is defined in Fig. } 13 \\
\text { and } \Delta X_{i}^{l} \text { enough far from } \\
\text { the growth interface. }\end{array}$ \\
\hline
\end{tabular}

(Received February 16, 2006)

(Revised April 6, 2006 (1st))

(Revised July 31, 2006 (2nd))

(Accepted August 8, 2006)

(Released February 28, 2007)

(Paper version of this article can be found in the IPSJ Transactions on Mathematical Modeling and Its Applications, Vol.48 No.SIG2(TOM16), pp.147-157.)

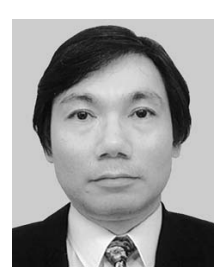

Hiromoto Susawa was born in 1964. He received his M.E. degree in Electrical and Computer Engineering from Nagoya Institute of Technology (N.I.T.) in 1988. In 1988, he joined Daido Steel Cooperation where he conducted research on thin films and semiconductors and filed patents. Since 1993, he has engaged in computational science. He received his M.E. degree in mechanical engineering from Daido Institute of Technology in 1995. Since 2004, he has been in doctoral studies at N.I.T. and studied simulation of Liquid Phase Epitaxy. He is a member of IPSJ, JSAP, JSME, SCEJ and VSJ.

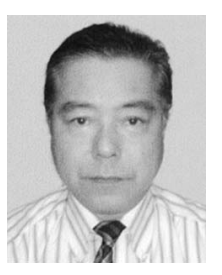

Toshihiro Tsuji was born in 1949. He received his M.E. and Ph.D. degrees from Kyushu University in 1973 and 1979, respectively. He has been working at Nagoya Institute of Technology since 1976 and is currently a professor in the graduate school. His research focuses on the areas of thermal and fluid engineering and polymer processing. He is a member of JSME, HTSJ, JSFM, JSPP and AIAA.

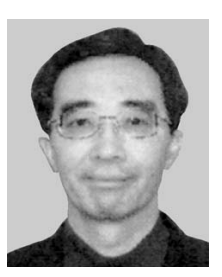

Kazumasa Hiramatsu was born in 1952. He received his M.E. and Ph.D. degrees from Nagoya University, Japan in 1977 and 1986, respectively. From 1983 to 1997, he worked at the Department of Electronics, Nagoya University, where he was engaged in the crystal growth of nitride semiconductors and LPE growth of III-V compound semiconductors. In 1997, he joined the Department of Electrical and Electronic Engineering at Mie University, continuing the study of the crystal growth of nitride semiconductors. He received the Patent of the Year in 2004. He is a member of JSPS, JACG, IEICE, MRS, APS and IEIJ.

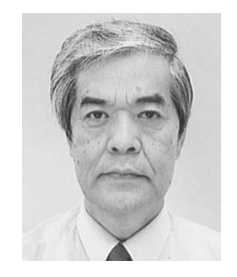

Takashi Jimbo was born in 1948. He received his B. Eng. and Dr. Eng. degrees from Nagoya University in 1970 and 1978, respectively. In 1975 , he joined Nagoya University and engaged in research on infrared lasers and detectors. Since 1987 he has been at the Nagoya Institute of Technology. He is currently a professor in the Research Center for Nano-Device and System, Nagoya Institute of Technology. He is a member of PSJ, JSAP, JSIR, LSJ, IEICE, IEEJ, JSMBE and SICE. 
Tetsuo Soga was born in 1959. He received his B.E. degree in 1982 from the Nagoya Institute of Technology, his M.E. degree in 1984 from the Nagoya Institute of Technology, and his Ph.D. degree in 1987 from

Nagoya University. He was appointed as a research associate in the Department of Electrical and Computer Engineering, Nagoya Institute of Technology in 1987. He is currently a professor in the Department of Environmental Technology, Nagoya Institute of Technology. 